CCC 2019

Proceedings of the Creative Construction Conference (2019) 006

Edited by: Miroslaw J. Skibniewski \& Miklos Hajdu

https://doi.org/10.3311/CCC2019-006

Creative Construction Conference 2019, CCC 2019, 29 June - 2 July 2019, Budapest, Hungary

\title{
Deep Learning-based Vehicle Image Matching for Flooding Damage Estimation
}

\author{
Somin Park ${ }^{\mathrm{a}}$, Francis Baek ${ }^{\mathrm{b}}$, Jiu Sohn ${ }^{\mathrm{a}}$, Hyoungkwan Kim* \\ ${ }^{a}$ School of Civil and Environmental Engineering, Yonsei Univ., 50 Yonsei-ro, Seodaemun-gu, Seoul 03722, Korea \\ ${ }^{b}$ Industrial Technical Laboratory, Yonsei Univ., 50 Yonsei-ro, Seodaemun-gu, Seoul 03722, Korea
}

\begin{abstract}
Images representing flooding damages can provide valuable information, such as the damage location and severity. Automated and quantifiable analyses of those images allow asset managers to accurately understand the vulnerability of the infrastructure. To this end, this paper proposes a methodology to match a vehicle in a flooding image to a 3D vehicle image. The proposed method is a part of a framework for flooding depth estimation. As the initial step of the framework, the proposed method uses Mask R-CNN and VGG network to extract the vehicle object and its features, respectively. The features of the vehicle images are compared with those of 3D vehicle image, to find a good match. A total of 87 vehicle objects were used to validate the proposed method, and promising levels of matching accuracy were obtained. Once the framework is completed, the proposed method is expected to automatically analyze flooding images for its damage assessment.
\end{abstract}

(C) 2019 The Authors. Published by Budapest University of Technology and Economics \& Diamond Congress Ltd.

Peer-review under responsibility of the scientific committee of the Creative Construction Conference 2019.

Keywords: Flooding damage; Vehicle image; 3D vehicle image; Image processing; Deep learning;

\section{Introduction}

Floods are one of the most dangerous and frequent natural disasters in the urban area, and have a great impact on road transportation. Road flooding due to heavy rain causes traffic accidents, traffic jam, and vehicle isolation. These disaster situations are often recorded by various types of cameras. CCTV (closed circuit television) cameras installed on the road or smart phone cameras carried by individuals can acquire photographs showing realistic disaster situations, and these images provide valuable information about the disaster. The ultimate objective of this study is to derive flooding damage information based on the image analysis of the vehicle, assuming that the vehicle is exposed to such a disaster situation. As a preliminary step to obtain the flood damage information, this paper proposes a method to match a vehicle in a flooding image to a $3 \mathrm{D}$ vehicle image. Once the matching is done accurately, the depth of the flooding would be able to be estimated.

*Corresponding author: Hyoungkwan Kim, email: hyoungkwan@yonsei.ac.kr 


\section{Methodology}

Figure 1 shows the proposed method with a focus on the matching of vehicle images. Photos of flooded roads are processed to segment only the vehicle objects, which are later compared with $3 \mathrm{D}$ vehicle images. In the method, two deep Convolutional Neural Networks (CNNs) are used. The first CNN is Mask R-CNN [1] for the vehicle image extraction. The second $\mathrm{CNN}$ is the VGG network [2] for the feature extraction. The similarity comparison is finally conducted to find the best matching between the two cross-domain images.

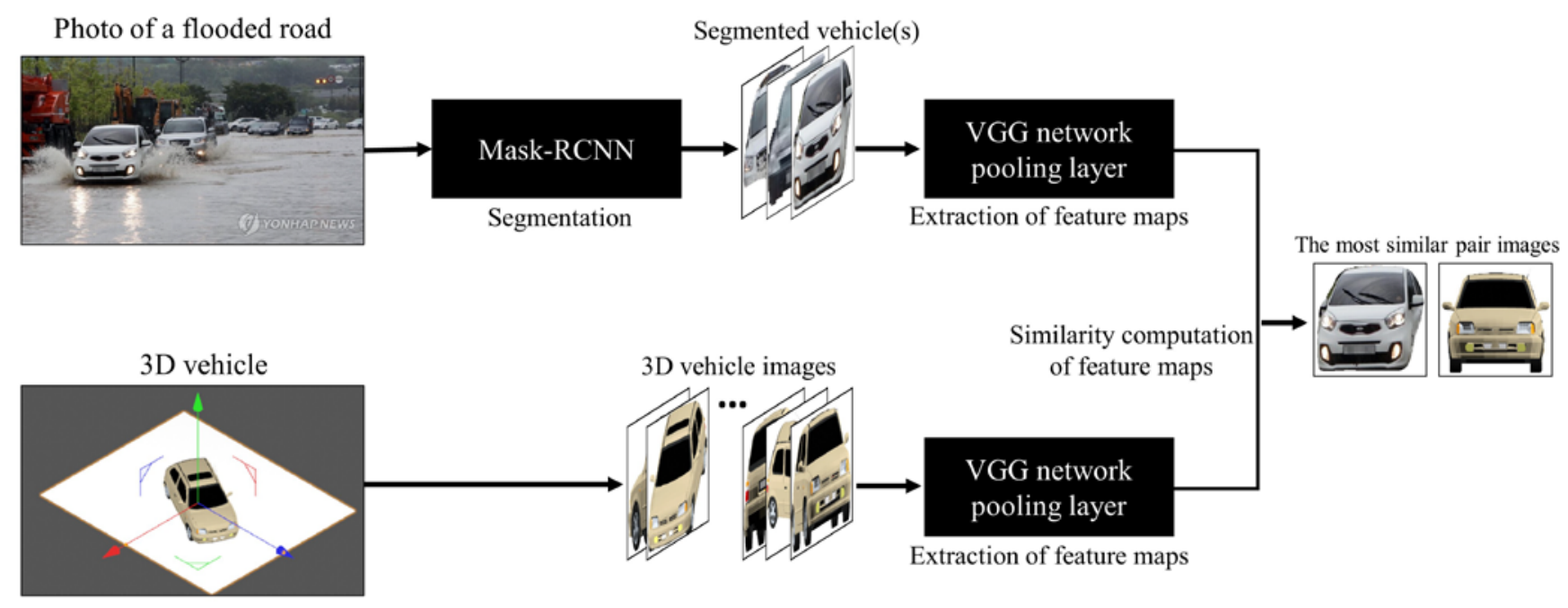

Fig. 1. The proposed method for matching a vehicle image and a 3D vehicle image.

\subsection{Segmentation}

Mask R-CNN is a deep learning model for object instance segmentation. Mask R-CNN extends Faster R-CNN [3] by including a mask branch for predicting segmentation masks [1]. Mask R-CNN were evaluated to be superior to previous approaches in the area of instance segmentation [1]. This paper, thus, used the Mask-RCNN pre-trained with the $80 \mathrm{k}$ images of COCO dataset [4].

\subsection{Similarity comparison}

Studies have been suggested to perform image retrieval using CNNs [5,6,7,8]. This paper used the method suggested by [8] for cross-domain image retrieval, in which the feature maps produced by the VGG network were vectorized and the vectorized feature maps were compared using the cosine distance. Feature maps are the output from a layer of a $\mathrm{CNN}$ and the input to the next layer of the CNN. Following the suggestions of [8], this study, for the comparison purpose, used the $4^{\text {th }}$ pooling layer of VGG16 and VGG19 [2].

\section{Experimental results}

\subsection{Data preparation}

The images of flooded vehicles were obtained from photographs of the floods of Korea in July and August 2018. In this way, 25 article photos containing vehicles were selected. The input of pre-trained Mask-RCNN was the article photos, and the output was the segmented vehicles. The number of vehicle objects obtained from the 25 photographs was 274. Except for those vehicles whose size was very small or overshadowed by other objects, 87 were used as data 
for the similarity evaluation. Figure 2 shows examples of excluded vehicle data. 3D vehicle images were obtained by rotating a 3D vehicle model by 10 degrees in two viewpoints (parallel and from slightly above). As a result, 72 vehicle images were created. Since the input size of VGG16 and VGG19 was fixed to 224 x 224, all image data used for similarity calculation were resized to that size.
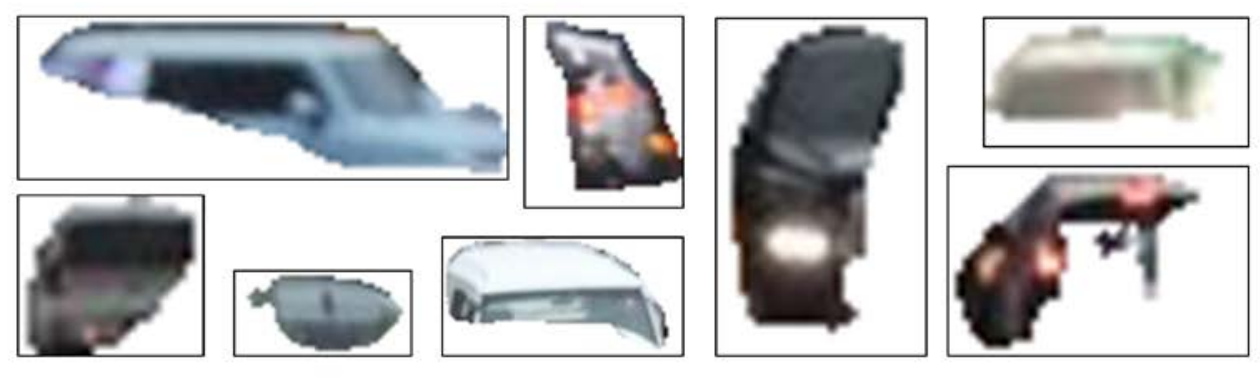

Fig. 2. Examples of excluded vehicle image.

\subsection{Evaluation standards}

As aforementioned, the $3 \mathrm{D}$ vehicle model was rotated by 10 degrees to acquire the data for image matching. Since a 3D model rotated by 10 degrees has similar visual characteristics to the image before rotation, the authors concluded that a difference in 10 degrees is negligible in the estimation of flood damage. Thus, the ground truth included a perfectly matched 3D image and a difference in rotation angle of 10 degrees to the left and right.

\subsection{Results}

Figure 3 shows examples of the matching results of the VGG16. As aforementioned, the fourth pooling layer of the VGG16 was used and the accuracy was 0.7356 . The VGG 19 showed a slightly worse performance with an accuracy of 0.6092 .
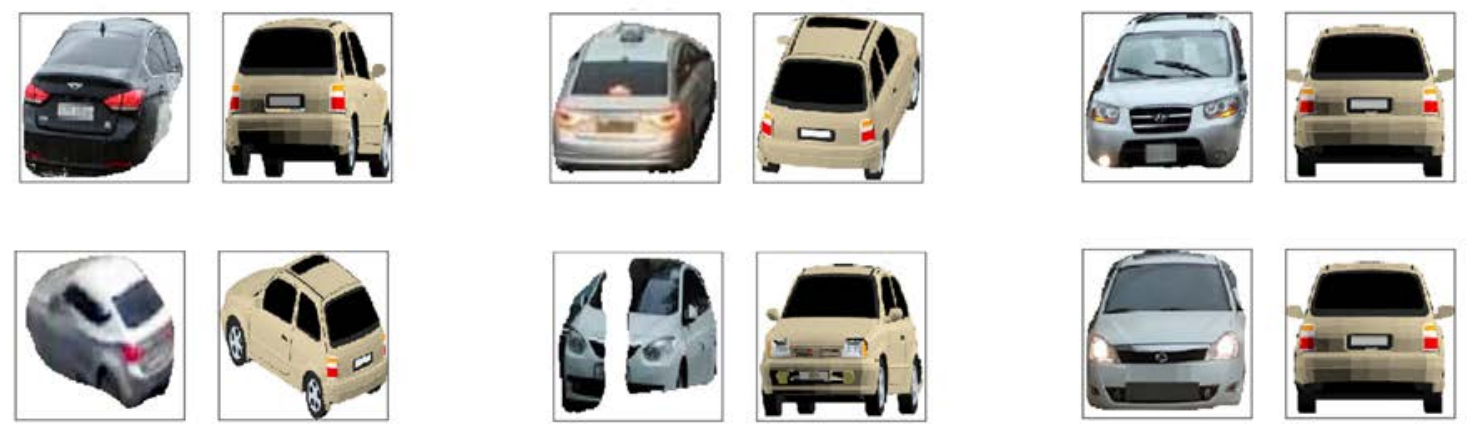

Fig. 3. Examples of the matching results of the VGG16.

\section{Discussion and conclusions}

In this study, 87 segmented vehicles from 25 article photos and 72 3D vehicle images were used as matching data. The 4th pooling layer of the VGG16 achieved better results than the 4th pooling layer of VGG19, with an accuracy of $73.56 \%$ and $60.92 \%$, respectively. As show in Fig. 4, segmented cars were in some cases represented as broken images because they were immersed in water or covered by other objects. However, even those broken images were able to be matched with $3 \mathrm{~d}$-images. This is because the feature maps of the 4th pooling layer of VGG extract structural characteristics rather than detailed characteristics [8]. 
This paper proposed a deep learning-based method to match between a vehicle in a flooding image and a 3D vehicle image. The proposed method was a part of a framework to automatically analyze images containing flooding situation. Mask R-CNN and VGG network were the major components of the proposed method, for vehicle extraction and feature extraction, respectively. The level of matching accuracy was promising for achieving the ultimate objective of the framework-the flooding depth estimation.

\section{Acknowledgements}

This work was supported by National Research Foundation of Korea (NRF) grants funded by the Ministry of Science and ICT (No. 2018R1A2B2008600) and the Ministry of Education (No. 2018R1A6A1A08025348).

\section{References}

[1] He, K., Gkioxari, G., Dollár, P., Girshick, R., Mask r-cnn, In Proceedings of the IEEE international conference on computer vision, 2017, pp. 2961-2969.

[2] Simonyan, K., Zisserman, A., Very deep convolutional networks for large-scale image recognition, arXiv preprint arXiv:1409.1556., 2014.

[3] Ren, S., He, K., Girshick, R., Sun, J., Faster r-cnn: Towards real-time object detection with region proposal networks, In Advances in neural information processing systems , 2015, pp. 91-99. https://doi.org/10.1109/tpami.2016.2577031

[4] Lin, T. Y., Maire, M., Belongie, S., Hays, J., Perona, P., Ramanan, D., Zitnick, C. L., Microsoft coco: Common objects in context, In European conference on computer vision, 2014, pp. 740-755.

[5] Zagoruyko, S., Komodakis, N., Learning to compare image patches via convolutional neural networks, In Proceedings of the IEEE conference on computer vision and pattern recognition, 2015,pp. 4353-4361. https://doi.org/10.1007/978-3-319-10602-1_48

[6] Radenovic, F., Tolias, G., Chum, O., Deep Shape Matching, In Proceedings of the European Conference on Computer Vision (ECCV), 2018, pp. 751-767. https://doi.org/10.1007/978-3-030-01228-1_46

[7] Appalaraju, S., Chaoji, V., Image similarity using deep CNN and curriculum learning, arXiv preprint arXiv:1709.08761., 2017.

[8] Ha, I., Kim, H., Park, S., \& Kim, H., Image retrieval using BIM and features from pretrained VGG network for indoor localization, Building and Environment, 2018, 140, pp. 23-31. 